\title{
RESEARCH OF NONLINEAR ELECTRIC CHAINS WITH TWO AND MORE NONLINEAR ELEMENTS IN SYSTEMS POWER SUPPLY
}

\author{
Burkhankhodzhayev A. M. ${ }^{1}$, Nurmatov B. $A^{2}$ \\ ${ }^{1}$ Tashkent state technical university, University street №2, Tashkent, 100095, Uzbekistan \\ 2 Tashkent state technical university, University street №2, Tashkent, 100095, Uzbekistan
}

\begin{abstract}
Work it is devoted to research of autoparametrical fluctuations at a frequency of the third subharmonica in nonlinear electric chains by two nonlinear elements. Conditions and a zone of steady excitement of a subharmonica of the third order, and also critical parameters the subharmonicas of the third providing steady excitement an order are defined.
\end{abstract}

\section{Introduction}

In the presents time development and introductions of effective and energy saving secondary power supplies for systems of automatic equipment, a sheaf is an actual task excitement and support of subharmonic fluctuations in nonlinear electric chains take a special place in the theory of nonlinear fluctuations and represent great theoretical and practical interest.

\section{Two and more nonlinear elements in systems power supply}

In the analysis of the mixed nonlinear electric chain consisting from two consistently connected parallel the ferrorezonansnykh of contours and eating from a source of tension (fig.1), as two connected fluctuations it is possible to take changes of streams $\boldsymbol{\psi}_{1}(\mathbf{t})$ and $\boldsymbol{\psi}_{2}(\mathbf{t})$ of two identical ferromagnetic elements.

At certain values of the $\boldsymbol{C}_{\mathbf{1}}, \boldsymbol{C}_{\mathbf{2}}$ and $\boldsymbol{g}_{\mathbf{1}}$ parameters, $\boldsymbol{g}_{\mathbf{2}}$ automodulation fluctuations at a frequency close to the frequency of a required subharmonica can be excited in each oscillatory contour [2].

In the course of automodulation fluctuations nonlinear inductance can "transform" energy of high frequency to energy of frequency of self-modulation. Counter inclusion of secondary windings of nonlinear induktivnost excludes penetrating the main harmonica in a secondary chain where only the subharmonica has to "be transformed". Introduction to the scheme of the additional selective contours which are adjusted on subharmonica frequency it is possible to improve the mode of division of frequency. The same purpose is carried out by the capacity of $\boldsymbol{C}_{\mathbf{3}}$ which with nonlinear inductance of an output contour forms; the resonant contour which is adjusted on the frequency of a required subharmonica.

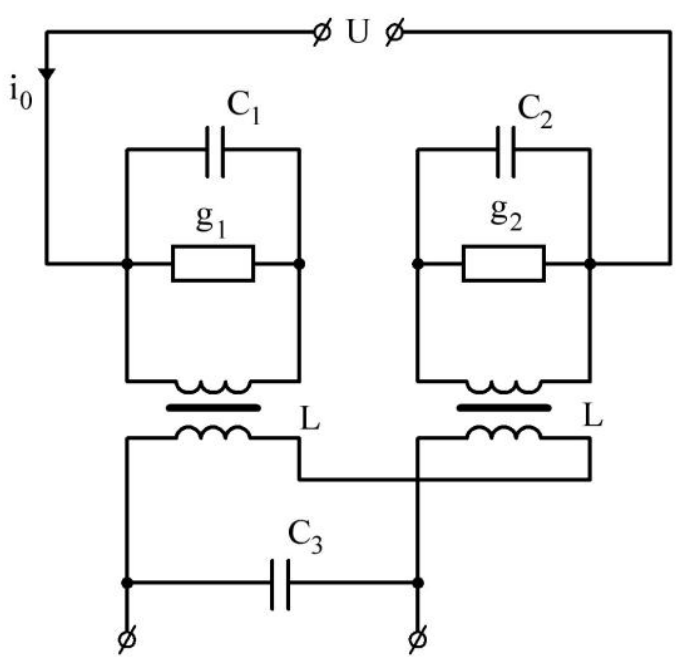

Fig. 1. Equivalent circuit converter 
For definition of a zone of steady initiation of subharmonic fluctuations in the studied chain we will work out the equations of electric balance:

$\left.\begin{array}{l}i_{L 1}+i_{C 1}+i_{g 1}=i_{L 2}+i_{C 2}+i_{g 2}=i_{0}(t), \\ U_{1}(t)+U_{2}(t)=U_{0}(t) .\end{array}\right\}$

If to accept approximation of communication between current $\boldsymbol{i}_{\boldsymbol{L}}$, and a stream $\boldsymbol{\psi}$ in a look $i_{L}=a \Psi+b \Psi^{3}$, instead of the first equation of system (1) it is possible to write down:

$$
\begin{aligned}
& C_{1} \frac{d^{2} \Psi_{1}}{d t^{2}}+g_{1} \frac{d \Psi_{1}}{d t}+a \Psi_{1}+b \Psi_{1}^{3}= \\
& =C_{2} \frac{d^{2} \Psi_{2}}{d t^{2}}+g_{2} \frac{d \Psi_{2}}{d t}+a \Psi_{1}+b \Psi_{1}^{3}=i_{0}(t)
\end{aligned}
$$

where, $\boldsymbol{\psi}_{1}$ and $\boldsymbol{\psi}_{2}$ - respectively instant values of flux linkage of nonlinear ferromagnetic elements;

$\mathbf{g}_{1}$ and $\mathbf{g}_{2}$ - active conductivity of contours, the considering losses in loadings and nonlinear coils (transformers);

$\mathbf{C}_{\mathbf{1}}$ and $\mathbf{C}_{2}$ - capacities of condensers the ferrorezonansnykh of contours.

Considering that in the mode of initiation of autoparametrical fluctuations by the most considerable on amplitudes the main harmonica and the chosen subharmonic component of streams $\boldsymbol{\psi}_{1}$ and $\boldsymbol{\psi}_{2}$ are, the solution of the differential equation of a chain can be set in a look:

$$
\left.\begin{array}{l}
\Psi_{1}=\Psi_{11} \sin \left(\omega t+\gamma_{1}\right)+\Psi_{1 / n} \sin (\omega t / n+\theta), \\
\Psi_{2}=\Psi_{21} \sin \left(\omega t+\gamma_{2}\right)+\Psi_{1 / n} \sin (\omega t / n+\theta) .
\end{array}\right\}
$$

where, $\psi_{11}$ and $\psi_{21}$ - amplitudes of streams of the main harmonica in cores of ferromagnetic elements of the corresponding contours;

$\boldsymbol{\psi}_{1 / \mathbf{n}}$ - order n-go subharmonica stream amplitude.

We will consider the mode of initiation of autoparametrical fluctuations at a frequency of the third subhannonica $(\boldsymbol{n}=\boldsymbol{3})$.

Substituting the estimated decision (3) in (2), after the simple transformations executed by method of harmonious balance for the main harmonica and a subharmonica of the third order we will receive:

$$
\begin{aligned}
& \left(-\beta_{1}+\Psi_{11}^{2}+2 \Psi_{1 / 3}^{2}\right) \Psi_{11}-\frac{1}{3} \Psi_{1 / 3}^{3} \cos \left(3 \theta-\gamma_{1}\right)= \\
& =\left(\beta_{2}+\Psi_{21}^{2}+2 \Psi_{1 / 3}^{2}\right) \Psi_{21}+\frac{1}{3} \Psi_{1 / 3}^{3} \cos \left(3 \theta-\gamma_{1}\right)
\end{aligned}
$$

$\alpha_{1} \Psi_{11}-\frac{1}{3} \Psi_{1 / 3}^{3} \sin \left(3 \theta-\gamma_{1}\right)=$

$=\alpha_{2} \Psi_{21}-\frac{1}{3} \Psi_{1 / 3}^{3} \sin \left(3 \theta-\gamma_{2}\right)$

$\left(-\beta_{1}^{\prime}+\Psi_{1 / 3}^{2}-2 \Psi_{11}^{2}\right) \Psi_{1 / 3}-$

$-\Psi_{11} \Psi_{1 / 3}^{2} \cos \left(\gamma_{1}-3 \theta\right)=$

$=\left(-\beta_{2}^{\prime}+\Psi_{1 / 3}^{2}-2 \Psi_{21}^{2}\right) \Psi_{1 / 3}-$

$-\Psi_{21} \Psi_{1 / 3}^{2} \cos \left(\gamma_{2}-3 \theta\right)$

$\alpha_{1}^{\prime} \Psi_{1 / 3}+\Psi_{11} \Psi_{1 / 3} \sin \left(\gamma_{1}-3 \theta\right)=$

$=\alpha_{2}^{\prime} \Psi_{1 / 3}+\Psi_{21} \Psi_{1 / 3} \sin \left(\gamma_{2}-3 \theta\right)$

Here,

$\beta_{1}=\frac{4\left(-C_{1} \omega^{2}+a\right)}{3 b} ; \beta_{2}=\frac{4\left(-C_{2}^{2} \omega^{2}+a\right)}{3 b} ;$

$\alpha_{1}=\frac{4 \omega g_{1}}{3 b} ; \alpha_{2}=\frac{4 \omega g_{2}}{3 b}$.

$\beta_{1}^{\prime}=\frac{4}{3 b} \cdot\left(-\frac{C_{1} \omega^{2}}{9}+a\right)$;

$\beta_{2}^{\prime}=\frac{4}{38} \cdot\left(-\frac{C_{2} \omega^{2}}{9}+a\right) ;$

$\alpha_{1}^{\prime}=\frac{4 \omega g_{1}}{9 b} ; \alpha_{2}^{\prime}=\frac{4 \omega g_{2}}{9 b}$.

The equations (4-7) are the equations of communication of a stream of the main harmonica and subharmonica of the third order and represent quite difficult dependence. For simplification of the analysis we will be limited to research of symmetric chains, i.e. we will accept:

$\beta_{1}=\beta_{2}=\beta ; \alpha_{1}=\alpha_{2}=\alpha ;$

$\beta_{1}^{\prime}=\beta_{2}^{\prime}=\beta^{\prime} ; \alpha_{1}^{\prime}=\alpha_{2}^{\prime}=\alpha^{\prime}$.

then we have:

$$
\begin{aligned}
& 2\left[-\beta^{\prime}+\Psi_{1 / 3}^{2}+\Psi_{11}^{2} \Psi_{21}^{2}\right]= \\
& =\left[\Psi_{1 / 3} \Psi_{11} \cos \left(\gamma_{1}-3 \theta\right)+\right. \\
& \left.+\Psi_{21} \cos \left(\gamma_{2}-3 \theta\right)\right], \\
& 2 \alpha^{\prime}=\Psi_{1 / 3}\left[\Psi_{11} \sin \left(\gamma_{1}-3 \theta\right)+\right. \\
& \left.+\Psi_{21} \sin \left(\gamma_{2}-3 \theta\right)\right] .
\end{aligned}
$$


Determination of tension of separate contours, the general current and other regime indicators of a chain at the set parameters of a source represents great theoretical and practical interest. Considering that:

$\Psi_{0 m} \cos \left(\omega t+\gamma_{0}\right)=\Psi_{11} \cos \left(\omega t+\gamma_{1}\right)=$

$=\Psi_{21} \cos \left(\omega t+\gamma_{2}\right)$

Here,

$\Psi_{0 m}=\frac{U_{0 m}}{\omega}$.

where, $U_{0 m}$ - amplitude value of tension of the power supply.

Having done simple transformations. we will receive:

$$
\left.\begin{array}{l}
\Psi_{01}^{2}=\Psi_{11}^{2}+\Psi_{21}^{2}+ \\
+2 \Psi_{11} \Psi_{21} \cos \left(\gamma_{1}-\gamma_{2}\right), \\
\Psi_{02}^{2}=\Psi_{11}^{2}+\Psi_{21}^{2}- \\
-2 \Psi_{11} \Psi_{21} \cos \left(\gamma_{1}-\gamma_{2}\right) .
\end{array}\right\}
$$

It is easy to show also that taking into account (10) equation (8) it is possible to copy in a look:

$$
\begin{aligned}
& Z_{1}^{2}+Z_{2}^{2}+4 Z+2 Z_{1} Z_{2}+4 Z_{1} Z+ \\
& +3 Z_{2} Z-4 \beta^{\prime}\left(Z_{1}+Z_{2}\right)-8 \beta^{\prime} Z+(11) \\
& +4\left(\alpha^{\prime 2}+\beta^{\prime 2}\right)=0
\end{aligned}
$$

Here,

$$
Z_{1}=\Psi_{01}^{2} ; Z_{2}=\Psi_{02}^{2} ; Z=\Psi_{1 / 3}^{2} \text {. }
$$

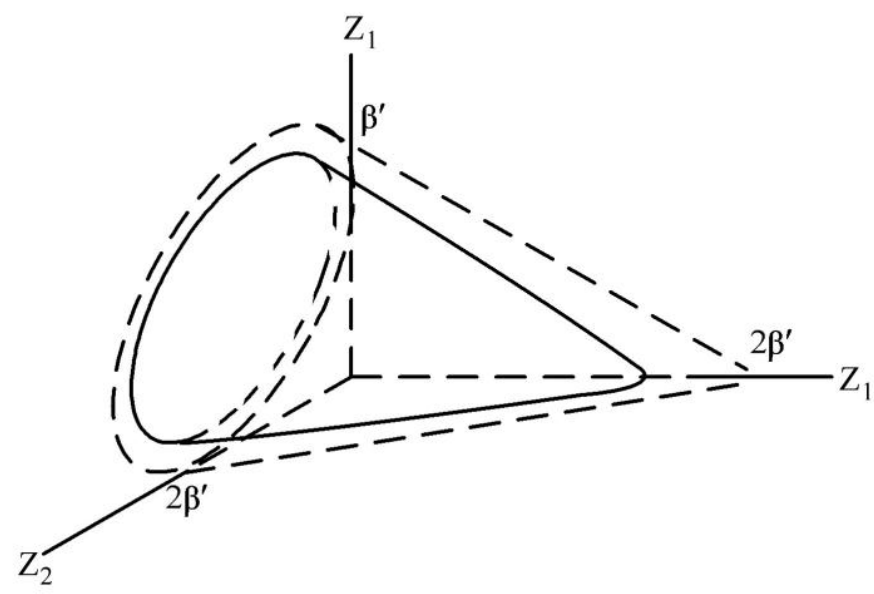

Fig. 2.

The equation (11) represents the equation of a two-way hyperboloid which invariants are equal:

$$
\begin{aligned}
& \Delta=-\left(\beta^{\prime 2}+\alpha^{\prime 2}\right)<0 ; S=6 ; \\
& T=20 ; \delta=0,25
\end{aligned}
$$

\section{Conclusion}

In space the part of a surface of a two-way hyperboloid describes a possible zone of steady existence of subharmonic fluctuations of the third order (fig. 2). More detailed data contain in $[1,3]$.

\section{References}

1. A.S.Karimov, G.R.Rakhimov, Auto-parametrical transformation of number of phases and frequency of alternating current, T.: Fan. 1975.
2. A.S.Karimov, M.T.Turdyev, D.Isamukhamedov, Single-phase ferromagnetic divider of frequency. Copyright certificate of the USSR, 678611., 1979 6U№29.

3. V.N.Ivashev, I.I.Parilis. Fluctuations in nonlinear electric systems. T.: Fan. 1967.

4. A.N.Miles, I.V.Volkov, Systems of invariable current on the basis of inductance-capacitor converters. - Kiev: Naukova thought, 1974, p. 216. 5. T.M.Kadyrov, H.A.Alimov, the Analysis of a double-circuit chain taking into account the highest harmonicas. - Izb. AN UZSSR, STN, 1979, №3., p.17-22. 